\title{
\begin{tabular}{l|} 
EXPERT \\
LEVIEWS \\
\hline
\end{tabular}
}

\section{Current controversies and challenges in allergic rhinitis management}

Expert Rev. Clin. Immunol. Early online, 1-13 (2015)

\section{David Price*, \\ Pete Smith, Peter Hellings, Nikos Papadopoulos, Wytske Fokkens, Antonella Muraro, Ruth Murray, Alison Chisholm, Pascal Demoly, Glenis Scadding, Joaquim Mullol, Phil Lieberman, Claus Bachert, Ralph Mösges, Dermot Ryan and Jean Bousquet}

*Author for correspondence: Tel.: +65 68097251 david@respiratoryresearch.org

For a full list of author affiliations, please see page 13.

There are many obstacles in the path of effective allergy management, in general, and allergic rhinitis (AR) control, in particular. Chief among them are: insufficient symptom relief in some patients provided by some currently considered first-line AR treatments in real life; an over-reliance on randomized controlled trials to direct AR guideline recommendations; the need for a broader interpretation of the AR evidence base (to include randomized controlled trials and real-life studies); poorly designed and interpreted studies; and lack of an AR control concept and common language of control. These controversies are fully reviewed here and challenging solutions have been presented.

\section{KEYwORDS: allergic rhinitis $\bullet$ clinical relevance $\bullet$ control $\bullet$ guidelines $\bullet$ randomized controlled trials, real life}

Allergic rhinitis (AR) is a global health problem, with an estimated prevalence of between 15 and $20 \%$ worldwide [1] which has been increasing. In other words, approximately 1 billion people from all corners of the globe, from all social and educational classes, young and old alike, suffer from this condition, with more people joining the AR rank every day. One might think with such strength of numbers that AR would be a major health priority for patients, doctors and governments, particularly when one considers that $\mathrm{AR}$ is a risk factor for asthma [2], the majority of rhinitis sufferers also have asthma [3] and that the presence of significant rhinitis increases the risk of poorly controlled asthma to the same extent as smoking [4]. The sad truth is that in reality, $\mathrm{AR}$ is virtually ignored; regarded as background noise, it is underestimated in terms of severity of symptoms and burden (both personal and economic) and, consequently, undertreated [5]. However, AR accounts for a very large socioeconomic burden [6], higher than that induced by type II diabetes and hypertension [7]. The knock-on cost is substantial, estimated at $€ 2$ billion in Sweden in terms of lost productivity alone [8].

The real face of $A R$ is that of a debilitating and an intrusive disease, debilitating in terms of its chronicity and intrusive in terms of its symptom burden and impact on patients' lives and the lives of their families [9]. At the height of the allergy season, patients with seasonal AR (SAR) suffer from symptoms approximately $50 \%$ of the time [10]. These symptoms can negatively impact every aspect of daily life including work, school and leisure activities and significantly impair patients' quality of life (QoL) [11]. Cognitive function can also be diminished due to poor sleep quality or use of sedating antihistamines, which is manifested in many ways including poorer school performance [12] and impaired driving performance (when untreated and symptomatic) [13]. Indeed, the magnitude of driving impairment is comparable to that seen at a blood alcohol level of $0.05 \%$, the legal limit in many countries [13].

Fortunately, the burden of AR (and all allergic diseases) is now being recognized at the EU level with the production of a written declaration on allergic disease. This declaration acknowledges the prevalence and burden of allergic disease, and calls on the European Commission and its member states to develop strategies to combat its impact [14]. It has been signed by 178 members of the European parliament. Signatories have taken the first important step in increasing their own awareness of $\mathrm{AR}$ and its impact, recognizing that $100 \mathrm{mil}$ lion Europeans suffer from AR and acknowledging the impact of this disease on the 
'social, professional and educational performance' of sufferers, particularly children, which leads to socioeconomic inequalities [14]. The Polish Presidency of the EU has also highlighted the importance of early diagnosis and management of allergic diseases to promote active and healthy aging [15], a priority of the EU [16,17]. Concurrently, the European Academy of Allergy and Clinical Immunology (EAACI) has launched an awareness campaign to 'help society to better understand how allergy sufferers feel, how profound allergy impacts QoL, how severe and costly the disease can become and how important it is to diagnose early and better manage this disease' [18]. Such initiatives are a first important step in the path of improving the lives of allergy sufferers around the world and a strong indication that at last allergy will be given the attention it deserves.

However, there are many obstacles in the path of effective allergy management, in general, and AR control, in particular. These controversies are reviewed fully here and challenging solutions have been presented.

\section{Controversies \& challenges \\ Controversy: changing face of AR \& insufficiency of monotherapy \& multiple therapy}

$\mathrm{AR}$, as a disease, is becoming ever more challenging to treat, with many factors contributing to this. In Europe, AR is now estimated to affect 100-150 million individuals, or nearly $25 \%$ of the population [18,19], and the prevalence continues to increase at a steady rate in many countries. Global climate changes resulting in elevated levels of greenhouse gases, such as carbon dioxide, increased industrialization, and increases in the quantities of airborne pollen and duration of pollen seasons may account, in part, for the increased prevalence [20-22]. Today, the majority of patients visiting their primary care physician have moderate/severe disease and persistent symptoms [11,23]. Patients are frequently poly-sensitized, making allergen avoidance problematic and persistent symptoms more likely [24]. The phenotype of the disease appears to be actively changing, with as many as $50-70 \%$ of patients suffering from mixed rhinitis (comprising both non-allergic rhinitis and AR) [25] and an estimated 10-20\% of patients affected by the treatment-resistant phenotype, that is, severe chronic upper airway disease [11,26]. Local AR, characterized as localized nasal allergic response in the absence of systemic atopy, has also been identified recently [27].

Patients' expectations from AR therapies are high [28]; at least $40 \%$ of rhinitis patients expect to be cured by the prescribed treatment [29]. Despite this high expectation, treatment satisfaction remains low, with up to two-thirds of AR patients reporting dissatisfaction with the results [30]. Although by following Allergic Rhinitis and its Impact on Asthma (ARIA) treatment recommendations, there is a significant reduction in disease severity among AR patients, up to $40 \%$ of patients remain in the moderate-severe category [31]. Other studies, however, have shown good response to second-generation antihistamines in a real-life setting, although the study had a non-comparative design [28].
The reasons for this dissatisfaction are complex. Changes in the complexity and severity of the disease (as described above) almost certainly contribute, and are most likely compounded by poor patient concordance with their AR therapy regimen, poor administration technique (for topically active medications), and also by the presence of co-morbid conditions such as asthma and more rarely by chronic rhinosinusitis. However, the most likely reason for dissatisfaction with treatment is that most AR treatment strategies, even in specialist care, are simply not effective enough, and do not provide adequate and rapid symptom relief [31,32]. Randomized controlled trials (RCTs) show mean effects, but many patients in real life remain symptomatic. The efficacy of intranasal corticosteroids (INS) has been shown to plateau, as recently evidenced by a failure to differentiate from placebo in providing complete/near-complete symptom relief in moderate/severe SAR patients [33]. The deficit of current AR therapy may be shown in several ways, directly by analyzing monotherapy treatment failure and indirectly by examining both physician co-prescribing behavior and patient-reported multiple therapy usage. For example, a historical cohort study, conducted with data from the Optimum Patient Care Research Database during the 2009-2010 pollen seasons in the UK, revealed that initial antihistamine or INS monotherapy failed in approximately $20-25 \%$ of SAR sufferers and $37-46 \%$ of perennial AR sufferers [34]. General practitioners in the UK prescribed multiple therapies as the first prescription of the season for many of their AR patients, but coprescription became more common as the season progressed, occurring in 45 and $>50 \%$ of SAR and perennial AR patients, respectively [34].

Studies have confirmed that use of multiple therapies (predominantly INS + oral antihistamines) is common and increasing [32,35], not only due to physicians' prescription but also as a consequence of the ready availability of AR treatments over the counter. However, multiple therapy has not proven to be effective [36]. The quantification of over-thecounter and online purchasing for rhinitis medications is an evolving challenge as it obscures the true burden of disease. The need for better and faster nasal and ocular symptom relief was cited by patients as the driver for this comedication behavior [32]. Despite multiple therapy usage, these patients reported significant residual nasal and ocular symptoms, a finding which has been replicated in other studies [37] and confirmed in the literature $[38,39]$. There is a need to simplify rhinitis treatment with a new single medication option which provides better and faster symptom control.

\section{Challenge: exceed efficacy of INS \& develop new treatments}

MP29-02 (Dymista ${ }^{\circledR}$; Meda AB, Solna, Sweden) is a new class of treatment for AR (WHO code ATC R01AD58), developed to have a rapid onset of action (which should encourage compliance), a sustained effect (to encourage continued regular use) and to induce better symptomatic relief than INS (to improve disease control and patient satisfaction). It delivers azelastine 

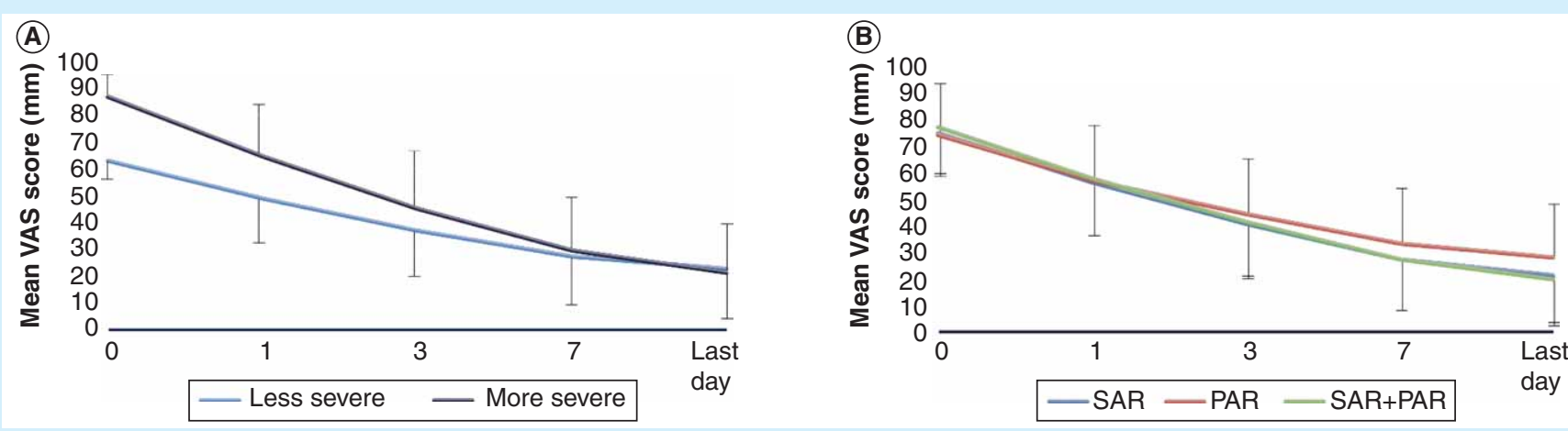

Figure 1. Effect of MP29-02 on VAS score over time according to (A) baseline severity and (B) allergic rhinitis phenotype. (A) Less severe - baseline VAS score of 50-74 mm $(n=641)$; more severe- baseline VAS score of 75-100 mm $(n=996)$. (B) SAR $(n=910)$; PAR $(n=179)$, SAR + PAR $(n=602)$. Data are presented as mean and standard deviation and are from a non-interventional real-life study in 1781 German allergic rhinitis patients with moderate/severe disease.

PAR: Perennial allergic rhinitis; SAR: Seasonal allergic rhinitis; VAS: Visual analog scale.

Reprinted with permission from Klimek et al. [41].

hydrochloride and fluticasone propionate together in a novel formulation and in a single device, and is indicated for the relief of symptoms of moderate/severe SAR and perennial AR, if monotherapy with either intranasal antihistamines or INS is not considered sufficient [40].

Patients treated with MP29-02 experienced twice the overall nasal and ocular symptom relief as those patients treated with either intranasal azelastine hydrochloride or fluticasone propionate, breaking the efficacy threshold of INS for the first time, once the benefit due to placebo was subtracted [33]. The benefits of MP29-02 were more pronounced in a real-life setting, with effectiveness established from the first day until treatment end, irrespective of severity or phenotype and with the feeling of well-controlled disease achieved for many patients after 3 days [41]. This real-life data is useful as it shows an even greater treatment effect than that observed in RCTs and in a way that is more clinically relevant (FIGURE 1). That said, there is a need for more comparative real-life data to elucidate the relative effectiveness of AR treatments.

At present, there are a number of other treatment options for AR in the pipeline. Several new formulations of existing drugs have been developed, including oral solutions of loratadine [42] and rupatadine [43], as well as a fast-dissolving tablet of ebastine. Other recently approved molecules such as ciclesonide and bilastine [44], as well as phosphodiesterase- 4 inhibitors have been proposed as treatments for AR. Ciclesonide has been tested in Phase IV trials and has demonstrated superiority over levocetirizine [45], while a Phase III trial of bilastine has demonstrated its superiority over cetirizine and desloratadine [46,47]; however, there are no recent data on the latter molecule. phosphoinositide 3-kinases inhibitors have undergone Phase I studies [NCT00836914], and prostaglandin receptor (CRTh2) antagonists have been associated with reduced airway inflammation [48]. $\mathrm{H} 3 / \mathrm{H} 4$ antagonists have recently been studied for the treatment of AR, but have not been shown to be more effective than $\mathrm{H} 1$ antagonists in reducing nasal congestion [49-51].
Omalizumab is an anti-IgE humanized monoclonal antibody that has been used to treat refractory asthma for over a decade and has been shown to improve QoL and relieve symptoms among patients with inadequately controlled AR [52]. In addition to conventional treatments, patients are also turning to alternative or complementary medicine for a variety of reasons including fear of the long-term consequences of medication and dissatisfaction with treatment outcomes [53]. Homeopathy has been associated with a modest improvement in symptoms and a reduction in conventional medication dosage, but a strong causal relationship has yet to be proven [54].

\section{Controversy: possible over-reliance on RCTs to guide $A R$ treatment recommendations}

The aim of any disease management guideline is to achieve control of that disease, and the AR management guidelines are no different $[55,56]$. Their function is to provide recommendations for physicians in frontline disease management on diagnosis, monitoring and treatment of real patients in a real-life setting. Paradoxically, the 'well' from which AR guideline committees draw the evidence to base their recommendations upon comes not from real life, but from the world of RCTs. Guidelines interpret scientific evidence to meet the needs of whole populations, with RCTs remaining the gold-standard evidence for assessing efficacy of treatment interventions. Levels of evidence are based on study design and the methodological quality of individual studies. Guideline developers must make a considered judgment about the generalizability, applicability, consistency and clinical impact of the evidence to create a clear link between the evidence and the recommendation. Grades of recommendation are based upon the strength of the supporting evidence, taking into account not only the overall level but also the considered judgment of the guideline developers. But what happens if the data source used by the guidelines is biased [57]?

Published data from RCTs (and meta-analyses of RCTs) are currently considered to be the 'gold-standard' evidence to 


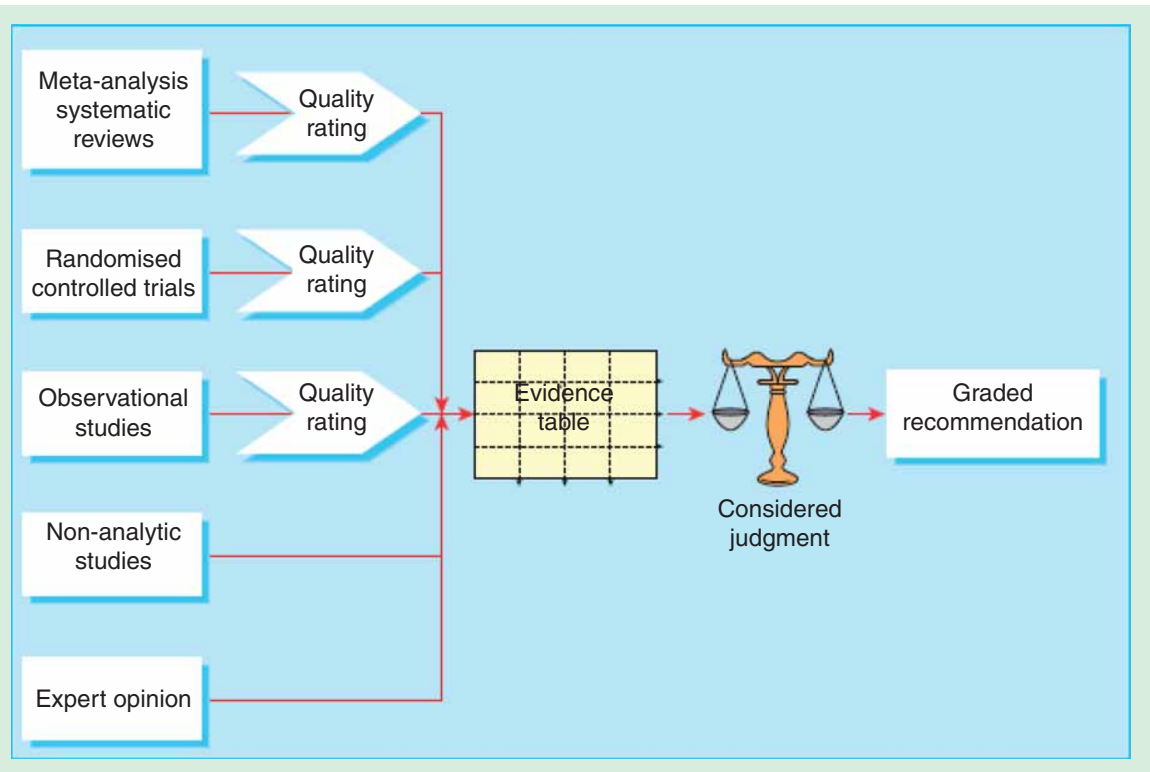

Figure 2. Overview of the process for developing and grading guideline recommendations.

Reproduced from 'A new system for grading recommendations in evidence based guidelines', R Harbour and J Miller, 323, 334-336, 2001 with permission from BMJ Publishing Group Ltd [58].

makers need to make careful judgments about the directness of the evidence from RCTs conducted in highly controlled settings'.

In addition to their strict inclusion and exclusion criteria, classical RCTs also benefit from multiple study supports that are not available in real life (e.g., free medication, high frequency of patient-physician interaction or follow-up, compliance checks/monitoring and use of complicated efficacy assessment tools) [59]. Such rigor and intensity of follow-up represents an ideal or optimized clinical situation which is rarely (if ever) replicated or feasible in everyday routine clinical practice. These controlled and optimized conditions enable RCTs to readily discern a direct cause-and-effect relationship between a particular trial intervention and an outcome. However, they do so at the expense of further reducing generalizability of RCT findings by increasing the 'distance' between RCT and real-life conditions. Poor external validity of RCTs is a partic-

inform treatment recommendations, placed firmly at the top of the evidence hierarchy (FIGURe 2) [58]. They are designed to assess efficacy of a particular intervention and to gather information on treatment-related adverse events. Primarily, RCTs are designed to address regulatory questions such as "can intervention "a" work in optimized clinical setting "b"?, rather than the more clinically pertinent question of, "can intervention "a" work in a variety of clinical settings "a," "b" and "c," in different patient populations, and what are the barriers and facilitators of its success?' While RCTs are well suited to address the first type of question by virtue of their high internal validity, they are less well suited to answer the second type of question due to their low external validity [59]. RCTs include a highly selected and homogenous patient population due to their strict inclusion and exclusion criteria. Patient recruitment into AR RCTs, for example, often requires patients to be non-smokers, to have no (or negligible) co-morbid disease or concurrent medication use, and to have good nasal inhaler technique and high adherence to study medication. Furthermore, patients are often required to have well-defined and characterized disease (e.g., a positive skin prick test to relevant pollen or other evidence of $\operatorname{IgE}$ sensitivity) and a certain severity of symptoms. Consequently, AR patients recruited into RCTs seldom represent the wider spectrum of patients seen in clinical practice, a point well made in a recently conducted prospective cohort study [60]. This study showed that of 311 AR patients seen by 48 general practitioners, only $7.4 \%$ would have been eligible for inclusion into one of four large placebo-controlled RCTs of persistent and intermittent AR [60]. Indeed, the authors went on to conclude that 'guideline developers and health decision ular concern for long-term chronic conditions, such as AR, that affect heterogeneous patient populations and are often associated with co-morbidity (e.g., asthma, sinusitis). Unfortunately, all of these RCT biases are incorporated into the evidence which informs the treatment guidelines. This problem is further confounded by the gold standard of meta-analysis, a statistical maneuver which is even further removed from real life.

\section{Challenge: consideration of RCT bias in treatment guideline recommendations}

RCTs (and meta-analyses) cannot (nor are they expected to) answer every clinical question. However, currently, they are the best way of assessing the size of effect, and are necessary not only for drug registration but also to establish general and objective evidence levels and recommendations. By design, they are not intended to answer questions about the effectiveness of interventions when used in less-controlled clinical management settings, but to answer tightly defined scientific questions while controlling as many variables as possible. The high cost associated with running RCTs also precludes their use for the investigation of long-term safety of an intervention and the assessment of rare treatmentrelated adverse events. Finally, RCTs offer little insight into intervention acceptability by patients and the practicalities and/or feasibilities of implementing new interventions into practice (including costs and cost-effectiveness considerations). Therefore, the key to addressing the gaps in the RCT evidence base is NOT to try and make the RCT into 'all things to all men', but rather to understand the question being asked and to answer that question using the study 
design most fit for purpose (i.e., RCT, pragmatic randomized trial or observational study).

Pragmatic trials are designed to reflect real patients and practice more closely than is typical of a classical RCT [59]. They compare interventions under more usual clinical circumstances. Patients included in such pragmatic trials are not subjected to the same rigors of inclusion and exclusion necessitated by RCTs, but are more heterogeneous in nature and more representative of patients seeking medical advice in everyday practice. Pragmatic trials also incorporate relevant levels of clinical care to help answer practical questions from all stakeholders involved in disease management; not only healthcare providers and patients, but also policy makers and insurers. Finally, pragmatic trials use stakeholder-centered outcome measures (e.g., patient-orientated measures for patients, cost-orientated measures for policy makers) to evaluate the effect of interventions and assess this effect over a time span appropriate to answer the question (which is often not feasible with an RCT). However, pragmatic trials are not without their own limitations. For example, as a consequence of lack of close monitoring, adequate patient follow-up in pragmatic trials can be an issue. Also, although they are less interventional than RCTs, the level of monitoring and patient engagement is in excess of that observed in real life, which may influence patient behavior and treatment effectiveness, and reduce patient participation [61].

Observational studies fall into one of three designs: cohort, case-control or cross-sectional design. These types of studies provide valuable data on how interventions are used and the results are associated with their use in real life [59]. They accomplish this goal in two ways: firstly, by not influencing or changing the normal patient-physician interaction in any way and secondly, by imposing no restrictions on the type of patient prescribed a given intervention, except that it is prescribed according to its summary of product characteristics. Real-life studies can detect strong associations between an intervention and a pre-defined outcome that are generalizable to a broad patient population. Data may be collected prospectively or drawn from retrospective datasets (e.g., electronic medical records, administrative claims databases), and as these data are routinely collected, results can often be obtained more quickly and at a lower cost than RCTs. As an intrinsic and unavoidable consequence of their design, observational studies possess lower internal validity than RCTs, limiting the extent to which they can demonstrate an unequivocal cause-and-effect relationship. For example, patients included in observational studies are not randomized, not blinded and missing data can limit interpretation of findings. However, bias can be minimized by identifying the eligible population, design, outcomes and potential confounding factors before the study commences and by using rigorous analytical methods.

The positioning of these trial designs relative to each other within the existing medical evidence framework, in terms of patient population and study conditions (or ecology of care), is shown graphically in Figure 3 [62]. The ecology of care $x$-axis uses a continuous scale to categorize studies from highly interventional and controlled RCTs at one end (with a constrained study design and rigorous follow-up) to observational studies at the other end (with a free ecology of care). Similarly, the population $y$-axis runs on a continuous scale from a highly selected narrow population at one end to a managed care broad patient population at the other end. This integrated view of the evidence base enables a clear visualization of the various study designs, their relationship to each other and their complementary nature. The different trial types can move (within limits) along each axis (as shown by the arrows) to position them in the 'best space' to answer a particular question.

There is no doubt that well-designed, conducted and analyzed RCTs are an integral and essential component in the guideline recommendation wheel. Used in isolation, RCTs lack the complementary components offered by pragmatic and observational studies, the inclusion of which improves the relevance of recommendations. Inclusion of pragmatic trials and observational studies is vital to support the evidence gathered 
from RCTs, as they can validate RCT findings in non-RCT populations and/or management settings or address questions unanswerable by the classical RCT design [62]. Thus, their findings can supplement (or complement) those from RCTs and help to qualify guideline recommendations. Sir Michael Rawlins (former chairman of NICE) recommended that one should consider all forms of evidence while taking into account the limitations and strengths of their respective designs [64]. Including evidence from observational and pragmatic studies and learning more about the effectiveness of interventions in patients actively excluded from RCTs could provide valuable insights into why many AR patients treated according to guideline-directed therapy remain symptomatic, with uncontrolled disease [11]. When conducting evidence reviews, guideline committees should consider both robustness of the data and its applicability. Evidence from effectiveness studies should be considered for inclusion to complement data already gathered from well-designed RCTs. However, in order to be considered, such real-life evidence needs to be of sufficiently high quality and also published. At the time of writing, an online search of Pubmed revealed that the number of observational studies (incorporating [allergic rhinitis] and [treatment] as search terms) was approximately $6 \%$ of the number of RCTs using the same search string. Clearly, there is some way to go in ensuring that observational and pragmatic studies are funded, conducted, published and of sufficiently high quality to be included (with RCTs) in the evidence used to inform guideline recommendations.

\section{Controversy: choosing the best way to grade scientific evidence}

At present, guideline recommendations are based primarily on evidence which is derived mostly, but not always, from RCTs, since there are few other reports. The evidence from these RCTs is graded according to the strength and robustness of the data. The best accepted methodology for grading scientific evidence for developing guidelines is the Grading of Recommendations Assessments, Development and Evaluation (GRADE) system [64-66], adopted by many organizations including WHO, Cochrane Collaboration, NICE and ARIA [55]. GRADE works by framing questions by patient/intervention/comparator/outcome, grading the evidence according to certain rules and providing quality-associated recommendations based on that grading (from weak to strong). The quality of evidence is graded as 'very low', 'low', 'moderate' or 'high', based on methodological quality and the likelihood of outcome bias. For the updated AR treatment guidelines [55], RCTs are automatically rated as high-quality evidence and observational studies as low-quality evidence, but these ratings can change. For example, factors that may decrease the quality include risk of bias, inconsistency, indirectness, imprecision and publication bias. Conversely, factors that may increase the quality include a large effect, dose response and minimization of confounding factors. GRADE offers a systematic way to evaluate scientific evidence, and provides answers to complex questions. However, it does suffer from some limitations. For example, it is not possible to address all clinical questions that may arise in everyday clinical practice. Also, the recommendations GRADE provides may not always be applicable in routine clinical care, especially since RCTs are carried out in highly selected patients who are not fully representative of the patient population consulting a physician. It is important to consider that RCT findings are limited in the extent to which they can be extrapolated to reflect treatment effects achievable at the population level [67]. Some expert bodies have chosen not to adopt it (e.g., most recommendations of Global Initiative for Asthma, Global initiative for chronic Obstructive Lung Disease, British Society of Allergy and Clinical Immunology) [68]. It is clear that some non-evidence-based analyses lead to recommendations which may be disputable and create confusion for physicians and patients.

\section{Challenge: call for a broader interpretation of the evidence base}

When interpreting the AR evidence base, it is important to consider not only what a particular treatment does (and the level or quality of evidence to support that) but also what it does not do and how to stratify patients so that they receive the appropriate treatment. A weak guideline recommendation may not allow physicians to easily select the most appropriate treatment course in a real-life setting. It is clear that some recommendations of ARIA GRADE are based on low-quality evidence, in particular, for the prevention of allergic diseases. This problem is not so apparent for treatment intervention recommendations. Thus, it is proposed for some low-evidence statements that healthcare providers and patients discuss the advantages and drawbacks of the therapies available. On the other end of the scale, an overinclusive (and strong) recommendation may over-grade the evidence, directing a particular course of treatment which may not be applicable to many patients seeking medical advice in routine care. Recommendations should not over-grade non-representative evidence from tightly controlled populations, but rather should clearly state who was studied and how they were managed. For example, INS are currently recommended by all AR guidelines $[55,56,69,70]$ as the most effective symptomatic AR treatment option in both adults and children, based on evidence from high-quality RCTs. Moreover, guidelines give an overall effect and patient stratification is not available. Real-life studies may, therefore, complement guidelines. Finally, guidelines may not be applicable to all patients, in all settings and in all care systems.

An integrated care pathway approach to AR management, recently proposed by Contre les MAladies Chronique pour un VIeillissement Actif (MACVIA) ARIA, is an important advancement [16], which empowers patients, encourages clinicians to exercise their own professional judgment, with a focus on quality and coordination of care, incorporating a mechanism for recording variations from planned care. The new MACVIA ARIA AR guideline based on a thorough GRADE evaluation will rank interventions from RCTs (including pragmatic trials), 
non-randomized studies of interventions as well as regulatory documents (i.e., summary of product characteristics and patient information leaflets) to create an evidence decision framework. These new guidelines will acknowledge that recommendations should be carefully considered with respect to availability, clinician and patient views, social determinants/barriers and cost/ reimbursement. The overall aim is to simplify AR management and guide treatment decisions in the real world.

\section{Controversy: many studies which inform the guidelines are poorly designed \& analyzed}

The design of RCTs stems from the regulatory rules under which they are governed [71]. For example, to achieve drug registration, RCTs are required to demonstrate non-inferiority of a new intervention versus a currently available option, and to be compared with the gold standard. However, interpreting the results of non-inferiority trials can be challenging. Where a superiority trial seeks to prove a clinically relevant difference between treatments (and is easy to interpret without further assumptions), a non-inferiority trial aims to show that a new intervention is no worse than a reference product (within predefined non-inferiority limits). Few registration trials are designed to assess superiority of a new intervention against the market leader, thus making it difficult for physicians and guideline committee members to differentiate one active treatment from another.

So, in order to achieve registration, RCTs are simply required to demonstrate a statistically significant difference between the drug and a placebo for the primary efficacy parameter. This poses the question: 'what does this outcome actually mean for patients and their physicians (?)', because in real life, patients do not take placebo. It is time that RCTs answered new questions, those which are relevant to patients and physicians alike. These may include: 'How much better will I feel compared to taking my previous therapy?' 'How soon will I feel this response?' 'Will I be symptom free?'

\section{Challenge: design better quality studies $\&$ assess data in a clinically relevant way}

Design better studies

Most trials comparing active drugs use non-inferiority methods; equivalence between fluticasone propionate twice daily and fluticasone furoate once a day was shown in this way [72]. However, superiority trials conducted against active comparators yield a higher quality of evidence that is more clinically relevant. The clinical development program of MP29-02 included such head-to-head comparisons versus marketed comparators [33] and, at the request of the US FDA, formulation- and devicematched comparators to negate the influence of formulation and device characteristics on clinical efficacy [73].

The efficacy of approved medications should be confirmed in real-life trials. MP29-02 has been assessed in a large multicenter, non-interventional study, which has established its effectiveness with a response greater than that seen in the RCTs and in all age groups from adolescents to the elderly [41].
Good-quality observational studies should reduce bias (selection, information, recall and detection) by defining hypotheses, objectives and analyses plans a priori [67]. All research components as well as study population and endpoint definitions should also be done prior to any analysis. For database studies, a suitable database should be identified or appropriate prospective data collection should be planned, ensuring that it contains adequate information and good-quality data from a sufficiently representative population [74]. A detailed database extraction and statistical analysis plan must be prepared. Possible confounders must be identified and accounted for by appropriately matching and/or adjustment strategies [75]. Observational studies should also be registered and the study protocol published in a public repository (if possible), with a commitment to publish regardless of the findings. In terms of analyses, it is of value to explore whether the study population is representative, to establish the consistency of results via sensitivity analyses and across relevant patient subgroups and to show reproducibility in different datasets (with similar criteria to define patient population and outcomes) [76,77].

\section{Respiratory effectiveness group}

The Respiratory Effectiveness Group (REG) is a not-for-profit, investigator-led initiative founded to endorse real-life research by establishing, applying and promoting quality standards against which the relevance, importance and utility of real-life data can be measured. The REG aims to do this by providing tools and methodologies to aid in the critical appraisal of data, and also to assess the quality and relevance of those data for clinical decision making and healthcare policy development. It has proposed a framework for categorizing all studies (RCTs, pragmatic trials and observational studies) in terms of management and care setting, and patient population. This approach acknowledges that studies can mirror real life in one or both of these domains and encourages partnership between different forms of data collection with the ultimate aim of making all studies produce stronger results in collaboration. The REG will lobby for the inclusion of real-life research into international guidelines by engaging with guideline bodies and research organizations as well as licensing authorities to ensure that reallife research is integrated into drug licensing and postmarketing appraisal processes.

The REG EAACI task force was established in 2014 with the stated aim of conducting a systematic critical review of real-life asthma literature between 2009 and 2013, and to report on the quality of real-life research in this period; highlighting studies meriting integration into asthma-related guidelines and policy decisions as well as recommending quality targets for the future. It serves as a vehicle through which REG can achieve its aforementioned goals, and also one through which EAACI can lead the critical appraisal and understanding of asthma-related pragmatic trials and observational studies. REG supports the development of tools and methodologies that permit quality assessment of real-life research. This initiative may be expanded to include AR in the future. 
Assess data in a clinically relevant way

At present, little is known as to what constitutes a clinically relevant response to AR treatment. A statistically significant response over placebo is confirmation of clinical efficacy, but assessing the relevance of this is a trickier proposition. Clinical relevance depends more on patient-related factors such as disease severity and predominant symptom, aspects that are not described by 'efficacy' alone. The EMA has proposed to define a clinically meaningful response using a responder analysis and the effect at $50 \%$ efficacy appears to mimic the clinical relevance [33,78]. So far, only one AR treatment (MP29-02) has been assessed in this way [33]. The efficacy of other AR treatments should be assessed by responder analysis to not only establish their clinical relevancy but also facilitate crosscomparisons.

\section{Controversy: lack of an AR control concept \& a common language of control}

It has been said that the single biggest problem in communication is the illusion that it has taken place. This appears to be particularly pertinent in the world of AR; numerous surveys have highlighted the mismatch between physician and patient expectations of, and experience with, AR treatments [29,30]. Part of the problem is that although the aim of AR treatment is to achieve disease control, there is a lack of consensus on what control actually is. The concept of control is well defined for other respiratory conditions such as asthma [79], and categorized as well controlled, partly controlled and uncontrolled. However, in AR and rhinosinusitis, the concept of control is very much in its infancy [26].

Physician-patient communication is also hampered by lack of a common language to describe AR control. The reflective total nasal symptom score and Rhinitis Quality of Life Questionnaire are routinely used in clinical trial settings to measure symptom severity and impact on QoL, respectively, but it is unclear what the scores (or change in scores) in these relative scales mean in terms of disease control. Responder analyses offer a more clinically relevant approach, but these are not routinely assessed in real life. Finally, several AR control questionnaires are available, such as Rhinitis Control Assessment Test [80], Allergic Rhinitis Control Test [81] and Control of Allergic Rhinitis and Asthma Test [82]. However, these surveys contain multiple questions and are not used routinely in clinical practice. In real life, physicians assess rhinitis control in many different ways, ranging from an assessment of symptom severity and frequency, activity impairment, rhinitis exacerbations to respiratory function [83].

\section{Challenge: improve communication in real life between key stakeholders}

A simple tool to both assess and define control is needed. A visual analog scale (VAS) is simple to use and intuitive to complete, making it ideal for everyday use. Although the VAS is a simple tool, it has been shown to correlate well with more complex AR outcome measures, such as the reflective total nasal symptom score and overall Rhinitis Quality of Life Questionnaire score [84], is sensitive enough to discriminate according to severity [85] and can assess the efficacy of AR treatments [86,87]. In real life, the VAS has been used to assess effectiveness of treatments [41] as well as inadequacies of others (including multiple treatments) [11]. ARIA in collaboration with MACVIA-LR (an EU reference site on active and healthy aging) has proposed a simple VAS to determine the level of control (using VAS score cut-offs) and to monitor symptom severity and effectiveness of treatment over time.

This VAS will be incorporated into the updated ARIA guideline, and used not only to assess disease control but also to direct treatment decisions as part of an integrated care pathway [16]. It will also form the central component of an app called Allergy Diary for AR patients, with a companion app introduced for both physicians and pharmacists to encourage better communication with patients and referral when appropriate. To use this VAS, respondents simply indicate their level of agreement or disagreement to the statement 'overall how much are your allergic symptoms bothering you today?' by placing a mark on a line between $0 \mathrm{~mm}$ (i.e., not at all bothersome) and $100 \mathrm{~mm}$ (extremely bothersome). A control cut-off for uncontrolled disease in AR has previously been defined as $50 \mathrm{~mm}$ [11]. The overall aim is to facilitate better communication between all stakeholders involved in AR (i.e., a common language of AR control), allow doctors to more easily comply with guidelines, better tailor AR treatment regimens to meet patients' needs and provide a tool that can be used in the controlled clinical trial world as well as in the real world.

\section{Expert commentary}

While the full treatment armamentarium for AR includes allergen immunotherapy and non-pharmacological interventions (e.g., nasal irrigation), this review is restricted to the challenges and controversies within the pharmacological management of AR. In particular, we have focused on the challenges that arise when the idealized therapeutic efficacy reported in classical RCTs interacts with aspects of the real world, and have discussed the challenges (and controversies) of assimilating different sources of evidence to underpin a more complete and informed approach to AR management.

There remain a number of outstanding research needs such as how best to target combination topical nasal treatment and whether topical antihistamines should be used more sparingly to avoid the associated systemic side effects. Moreover, questions exist around the potential role of allergen immunotherapy in the treatment of patients with both allergic and non-allergic components to their rhinitis.

The necessary trials will take some time to be designed and conducted, but in the short term, simple tools are available (and are in development) that have practical utility and important implications for routine care. Among allergists, for example, there is a growing call to set aside complex assessments 
and, instead, return to more easily implementable tools with equal utility tools such as VAS, as operationalized in the recently launched MACVIA ARIA initiative. In parallel, the EAACI in conjunction with the REG [88] are developing a simple checklist to support the evaluation of observational studies, in order to enable authors to publish and guideline groups to identify real-life evidence that can, and should, be used to inform future clinical practice. Moreover, when appraising RCT evidence, guideline groups must also start to consider (and record) the applicability of RCTs to routine care, in terms of both ecology of care and the patient population. Only by including real-life evidence in our clinical thinking can we return the patient to the center of our care planning and, together, chart a path toward more tailored therapeutic management in AR and improved outcomes.

\section{Five-year view}

Partnering of question with study design should develop in the future. Key stakeholders (e.g., physicians, guideline committees, pharmaceutical companies and payers) should carefully consider the question which they would like to answer and select the most appropriate study design suited to the task. RCTs will remain the gold-standard tool to assess efficacy in a controlled setting, but other study designs (e.g., pragmatic and observational studies) should be used to complement evidence obtained in RCTs. For example, payers frequently request reallife data to guide their decisions. Real-life studies are a necessary part of the totality of the evidence base, but work must continue to ensure that they are of high quality. A very simple real-life study quality appraisal tool is required, and this is currently being developed at Research in Real Life in conjunction with an EAACI Task Force.

Precision in study result assessment is also necessary, and this should be encouraged in the future. A clear understanding of what a particular study shows, and perhaps more importantly what it does not show, is missing. Data should also be assessed in a way that is clinically relevant to patients and physicians. Real-life data inherently does that. Advancements have recently been made in assessment of AR RCT data in a more clinically relevant way (e.g., via responder analyses) [33]. Existing and novel AR treatments should be compared using this clinically relevant approach.

Finally, AR guidelines are essentially designed to assist clinicians in diagnosing, treating and ultimately achieving (and maintaining) control of the disease. Although they are widely disseminated around the world, they are (unfortunately) little implemented because they are too complex. A simpler approach to AR management is needed. MACVIA ARIA is leading the way in this regard with a proposal for an AR integrated care pathway which encourages patient empowerment and physician engagement, so that patients and physicians are guided and not led. A clinical decision support system using a simple VAS to direct treatment decision is also under development and should revolutionize the way AR is managed in real life.

\section{Financial \& competing interests disclosure}

This paper has been supported by an unrestricted educational grant from Meda. D Price has board membership with Aerocrine, Almirall, Amgen, AstraZeneca, Boehringer Ingelheim, Chiesi, Meda, Mundipharma, Napp, Novartis and Teva. Consultancy: A Almirall, Amgen, AstraZeneca, Boehringer Ingelheim, Chiesi, GlaxoSmithKline, Meda, Mundipharma, Napp, Novartis, Pfizer and Teva; grants and unrestricted funding for investigator-initiated studies from UK National Health Service, British Lung Foundation, Aerocrine, AKL Ltd, Almirall, AstraZeneca, Boehringer Ingelheim, Chiesi, Eli Lilly, GlaxoSmithKline, Meda, Merck, Mundipharma, Napp, Novartis, Orion, Pfizer, Respiratory Effectiveness Group, Takeda, Teva and Zentiva; payments for lectures/ speaking: Almirall, AstraZeneca, Boehringer Ingelheim, Chiesi, Cipla, GlaxoSmithKline, Kyorin, Meda, Merck, Mundipharma, Novartis, Pfizer, SkyePharma, Takeda and Teva; payment for manuscript preparation: Mundipharma and Teva; patents (planned, pending or issued): AKL Ltd; payment for the development of educational materials: GlaxoSmithKline, Novartis; stock/stock options: shares in AKL Ltd which produces phytopharmaceuticals and owns $80 \%$ of Research in Real Life Ltd and its subsidiary social enterprise Optimum Patient Care; received payment for travellaccommodations/meeting expenses from Aerocrine, Boehringer Ingelheim, Mundipharma, Napp, Novartis and Teva; funding for patient enrollment or completion of research: Almirral, Chiesi, Teva and Zentiva; and peer reviewer for grant committees: Medical Research Council (2014), Efficacy and Mechanism Evaluation program (2012), HTA (2014). P Smith has received honoraria for GSK, MSD, Mundipharma and Meda. P Hellings is the recipient of unrestricted research grants of Meda, Stallergenes and ALK. N Papadopoulos has received grants from GSK, Nestle and Merck; provided consultancy to GSK, Abbvie, Novartis, Menarini, Meda and Alk-Abello; is on the speakers bureau for Novartis, Allegopharma, Uriach, GSK, Stallergenes and MSD; and has provided educational presentations for Abbvie, Sanofi and Meda. W Fokkens has received an educational grant from $M E D A$ and research grants from GSK, Biopharma. A Muraro has lectured for Meda, Nutricia, Novartis, Allergopharma; was the Secretary General of EAACI in 2013-2015 and the President of EAACI from 2015 to 2017. R Murray is the Director of a Medical and Scientific Affairs consultancy, who has provided consultancy services to Meda, MACVIA ARIA and RIRL. P Demoly is a consultant (and a speaker) for Stallergenes, Circassia, ALK, DBV and Chiesi, and was a speaker for Merck, Astra Zeneca, Pierre Fabre Médicaments, Menarini, Allergopharma, Allergy Therapeutics Ltd, Thermo Fischer Scientific and GlaxoSmithKline. G Scadding has received research grants from GSK and $A L K$, as well as honoraria for articles, consulting, lectures/chairing and/ or advisory boards from ALK, Bausch \& Lomb, Church \& Dwight, Circassia, GSK, Groupo Uriach, Meda, Merck, Ono, Shionogi and Stallergenes. J Mullol is or has been a member of national and international scientific advisory boards (consulting), received fees for lectures, and grants for research projects from ALK-Abelló, Boehringer Ingelheim, Crucell, Esteve, FAES, GSK, Hartington Pharmaceuticals, Johnson \& Johnson, Meda Pharma, MSD, Novartis, Pierre Fabre, Sanofi-Aventis, Schering Plough, UCB, Uriach Group and Zambon. P Lieberman is a speaker for Meda, Teva, Merck, Genentech, Mylan, and a consultant for Sanofi, Novartis, Genentech and Mylan. C Bachert is on the speaker's bureau for Meda. R Mösges receives personal fees, or grants from 
ALK-Abello, Allergy Therapeutics, Allergopharma, Bencard, BiotechTools, Bayer, GSK, HAL, Johnson + Johnson, Lofarma, MSD, Menarini, Faes, Novartis, Leti, Optima, AIPrevent, Servier, Stada, Stallergenes, UCB, Ursapharm, Bitop, Hulka, Arthrocare, Meda and Ohropax. He has received non-financial support from Greer, Roxall, UCB and Atmos. Outside the submitted work, Ralph Möges is a member of the guidelines task force of the German Academy of Otorhinolaryngology. He is the chairman of ISCOANA, the International Standardization Committee of the European Rhinologic Society (ERS), the Chairman of the ENT-section of the European Academy of Allergy, Asthma and Clinical Immunology (EAACI) and a Vice-president of INTERASMA. D Ryan is paid consultancy fees by Stallergenes, Uriach and Teva. He has lectured on behalf of Meda, GSK, AZ, Chiesi, Thermo Fisher, Boehringer, Novartis and Almirall. He is the Director of Health Strategy at Optimum Patient Care. J Bousquet has received honoraria for scientific and advisory boards: Almirall, Meda, Merck, MSD, Novartis, Sanofi-Aventis, Takeda, Teva, Uriach; lectures during meetings for the following: Almirall, AstraZeneca, Chiesi, GSK, Meda, Menarini, Merck, MSD, Novartis, Sanofi-Aventis, Takeda, Teva, Uriach; and is on the Board of Directors for Stallergenes. The authors have no other relevant affiliations or financial involvement with any organization or entity with a financial interest in or financial conflict with the subject matter or materials discussed in the manuscript apart from those disclosed.

\section{Key issues}

- Controversy 1: Allergic rhinitis (AR) is becoming ever more challenging to treat, with intranasal corticosteroids providing insufficient symptom relief for many patients.

- Challenge 1: There is a need for new AR treatment options which provide faster and more complete symptom relief than intranasal corticosteroids.

- Controversy 2: AR treatment guidelines rely predominantly on evidence generated from randomized controlled trials (RCTs). Currently, RCTs are the best way of assessing size of a treatment effect and are necessary not only for drug registration but also to establish general and objective evidence levels and recommendations. However, they cannot (nor are they expected to) answer every clinical question.

- Challenge 2: High-quality evidence from effectiveness studies should be considered for inclusion in guidelines to complement data already gathered from well-designed RCTs.

- Controversy 3: Currently, AR management guidelines grade the evidence in such a way that they may not always be readily transferable to routine clinical care.

- Challenge 3: A simplified approach to guide treatment decisions in the real world is needed.

- Controversy 4: Many studies which inform the guidelines are poorly designed and analyzed.

- Challenge 4: There is an urgent need for good-quality studies in AR, both RCT and observational. Data from these studies should be analyzed and interpreted in a way which makes sense to physicians and patients and also to guideline committee members.

- Controversy 5: AR control remains the aim of current management guidelines, yet currently a control concept and control language are lacking in AR.

- Challenge 5: A simple visual analog scale has been advocated by Contre les MAladies Chronique pour un Vleillissement Actif Allergic Rhinitis and its Impact on Asthma as the new language of AR control and the control cut-offs have been defined. This visual analog scale will be incorporated into the new AR management guideline called the AR integrated care pathway.

\section{References}

Papers of special note have been highlighted as:

- of interest

•• of considerable interest

1. Mygind N. Allergic rhinitis. Chem Immunol Allergy 2014;100:62-8

2. Shaaban R, Zureik M, Soussan D, et al. Rhinitis and onset of asthma: a longitudinal population-based study. Lancet 2008;372: $1049-57$

3. Khan DA. Allergic rhinitis and asthma: epidemiology and common pathophysiology. Allergy Asthma Proc 2014;35:357-61

4. Clatworthy J, Price D, Ryan D, et al. The value of self-report assessment of adherence, rhinitis and smoking in relation to asthma control. Prim Care Respir J 2009;18:300-5

5. Ruokonen M, Kaila M, Haataja R, et al. Allergic rhinitis in school-aged children with asthma - still under-diagnosed and undertreated? A retrospective study in a children's hospital. Pediatr Allergy Immunol 2010;21: e149-54

6. Zuberbier T, Lotvall J, Simoens S, et al. Economic burden of inadequate management of allergic diseases in the European Union: a GA(2) LEN review. Allergy 2014;69:1275-9

7. de la Hoz CB, Rodriguez M, Fraj J, et al. Allergic rhinitis and its impact on work productivity in primary care practice and a comparison with other common diseases: the Cross-sectional study to evAluate work Productivity in allergic Rhinitis compared with other common dIseases (CAPRI) study. Am J Rhinol Allergy 2012;26:390-4

8. Hellgren J, Cervin A, Nordling S, et al. Allergic rhinitis and the common cold-high cost to society. Allergy 2010;65:776-83

9. Bellanti JA, Settipane RA. The burden of allergic rhinitis on patients' quality of life. Allergy Asthma Proc 2012;33(Suppl 1): S112

10. Small M, Piercy J, Demoly P, Marsden H. Burden of illness and quality of life in patients being treated for seasonal allergic 
rhinitis: a cohort survey. Clin Transl Allergy 2013;3:33

11. Bousquet PJ, Bachert C, Canonica GW, et al. Uncontrolled allergic rhinitis during treatment and its impact on quality of life: a cluster randomized trial. J Allergy Clin Immunol 2010;126:666-8

12. Jauregui I, Mullol J, Davila I, et al. Allergic rhinitis and school performance. J Investig Allergol Clin Immunol 2009;19(Suppl 1): 32-9

13. Vuurman EF, Vuurman LL, Lutgens I, Kremer B. Allergic rhinitis is a risk factor for traffic safety. Allergy 2014;69:906-12

14. Antonescu E, Childres N, Gardini E, et al. Written declaration on recognising the burden of allergic disease. European Parliament 2014. [Epub ahead print]

15. Samolinski B, Fronczak A, Kuna P, et al. Prevention and control of childhood asthma and allergy in the EU from the public health point of view: Polish Presidency of the European Union. Allergy 2012;67: 726-31

16. Bousquet J, Addis A, Adcock I, et al. Integrated care pathways for airway diseases (AIRWAYS-ICPs). Eur Respir J 2014;44: 304-23

17. Bousquet J, Michel J, Standberg T, et al. The european innovation partnership on active and healthy aging: the european geriatric medicine introduces the EIP on AHA column. Eur Geriatr Med 2014;5: 361-2

18. European academy of allergy and clinical immunology (EAACI). Beware of allergy campaign. 2015. Available from: www. bewareofallergy.com/ [Last accessed 4 April 15]

19. Calderon MA, Demoly P, Gerth van WR, et al. EAACI: A european declaration on immunotherapy. designing the future of allergen specific immunotherapy. Clin Transl Allergy 2012;2:20

20. Levetin E, Van de Water P. Changing pollen types/concentrations/distribution in the United States: fact or fiction? Curr Allergy Asthma Rep 2008;8:418-24

21. Bartra J, Mullol J, del CA, et al. Air pollution and allergens. J Investig Allergol Clin Immunol 2007;17(Suppl 2):3-8

22. Davila I, Mullol J, Bartra J, et al. Effect of pollutants upon patients with respiratory allergies. J Investig Allergol Clin Immunol 2007;17(Suppl 2):9-20

23. Demoly P, Bousquet PJ, Mesbah K, et al. Visual analogue scale in patients treated for allergic rhinitis: an observational prospective study in primary care: asthma and rhinitis. Clin Exp Allergy 2013;43:881-8

24. Ciprandi G, Cirillo I. Monosensitization and polysensitization in allergic rhinitis. Eur J Intern Med 2011;22:e75-9

25. Bernstein JA. Allergic and mixed rhinitis: Epidemiology and natural history. Allergy Asthma Proc 2010;31:365-9

26. Hellings PW, Fokkens WJ, Akdis C, et al. Uncontrolled allergic rhinitis and chronic rhinosinusitis: where do we stand today? Allergy 2013;68:1-7

27. Rondon C, Campo P, Zambonino MA, et al. Follow-up study in local allergic rhinitis shows a consistent entity not evolving to systemic allergic rhinitis. J Allergy Clin Immunol 2014;133:1026-31

28. Demoly P, Chiriac AM, Berge B, Rostin M. Reasons for prescribing second generation antihistamines to treat allergic rhinitis in real-life conditions and patient response. Allergy Asthma Clin Immunol 2014;10:29

29. Hellings PW, Dobbels F, Denhaerynck K, et al. Explorative study on patient's perceived knowledge level, expectations, preferences and fear of side effects for treatment for allergic rhinitis. Clin Transl Allergy 2012;2:9

30. Ciprandi G, Incorvaia C, Scurati S, et al. Patient-related factors in rhinitis and asthma: the satisfaction with allergy treatment survey. Curr Med Res Opin 2011;27:1005-11

31. Mullol J, Bartra J, del CA, et al. Specialist-based treatment reduces the severity of allergic rhinitis. Clin Exp Allergy 2013;43:723-9

32. Pitman R, Paracha N, Parker C, et al. Episode pattern and healthcare utilisation in patients with seasonal allergic rhinitis. Allergy 2012;67:A885

33. Meltzer E, Ratner P, Bachert C, et al. Clinically relevant effect of a new intranasal therapy (MP29-02) in allergic rhinitis assessed by responder analysis. Int Arch Allergy Immunol 2013;161:369-77

- This reference is of interest as it recommends analyzing data in a more clinically relevant way.

34. Price D, Scadding G, Bachert C, et al. Monotherapy failure and UK co-prescribing practices: proxy markers of unmet need in allergic rhinitis. Prim Care Res J 2015; submitted

35. Navarro A, Valero A, Rosales MJ, Mullol J. Clinical use of oral antihistamines and intranasal corticosteroids in patients with allergic rhinitis. J Investig Allergol Clin Immunol 2011;21:363-9

36. Anolik R; Mometasone Furoate Nasal Spray With Loratadine Study Group. Clinical benefits of combination treatment with mometasone furoate nasal spray and loratadine vs monotherapy with mometasone furoate in the treatment of seasonal allergic rhinitis. Ann Allergy Asthma Immunol 2008;100:264-71

37. Bousquet PJ, Demoly P, Devillier P, et al. Impact of allergic rhinitis symptoms on quality of life in primary care. Int Arch Allergy Immunol 2013;160:393-400

38. Esteitie R, deTineo M, Naclerio RM, Baroody FM. Effect of the addition of montelukast to fluticasone propionate for the treatment of perennial allergic rhinitis. Ann Allergy Asthma Immunol 2010;105: 155-61

39. $\mathrm{Kim} \mathrm{CH}, \mathrm{Kim} \mathrm{JK}, \mathrm{Kim} \mathrm{HJ}$, et al. Comparison of intranasal ciclesonide, oral levocetirizine, and combination treatment for allergic rhinitis. Allergy Asthma Immunol Res 2015;7:158-66

40. Dymista summary of product characteristics. Available from: www.medicines.orguk/emc/ medicine/27579 2014

41. Klimek L, Bachert C, Mosges R, et al. Effectiveness of MP29-02 for the treatment of allergic rhinitis in real-life: results from a noninterventional study. Allergy Asthma Proc 2015;36:40-7

42. Wu KG, Li TH, Wang TY, et al. A comparative study of loratadine syrup and cyproheptadine HCL solution for treating perennial allergic rhinitis in Taiwanese children aged $2-12$ years. Int J Immunopathol Pharmacol 2012;25:231-7

43. Potter P, Maspero JF, Vermeulen J, et al. Rupatadine oral solution in children with persistent allergic rhinitis: A randomized, double-blind, placebo-controlled study. Pediatr Allergy Immunol 2013;24:144-50

44. Bousquet J, Ansotegui I, Canonica GW, et al. Establishing the place in therapy of bilastine in the treatment of allergic rhinitis according to ARIA: evidence review. Curr Med Res Opin 2012;28:131-9

45. Kim CH, Kim JK, Kim HJ, et al. Comparison of intranasal ciclesonide, oral levocetirizine, and combination treatment for allergic rhinitis. Allergy Asthma Immunol Res 2015;7:158-66

46. Kuna $P$, Bachert $C$, Nowacki Z, et al. Efficacy and safety of bilastine $20 \mathrm{mg}$ compared with cetirizine $10 \mathrm{mg}$ and placebo for the symptomatic treatment of seasonal allergic rhinitis: a randomized, double-blind, 
parallel-group study. Clin Exp Allergy 2009;39:1338-47

47. Bachert C, Kuna P, Sanquer F, et al. Comparison of the efficacy and safety of bilastine $20 \mathrm{mg}$ vs desloratadine $5 \mathrm{mg}$ in seasonal allergic rhinitis patients. Allergy 2009;64:158-65

48. Pettipher R, Hansel TT. Antagonists of the prostaglandin D2 receptor CRTH2. Drug News Perspect 2008;21:317-22

49. Lazewska D, Kiec-Kononowicz K. Azines as histamine $\mathrm{H} 4$ receptor antagonists. Front Biosci (Schol Ed) 2012;4:967-87

50. Daley-Yates P, Ambery C, Sweeney L, et al The efficacy and tolerability of two novel $\mathrm{H}$ (1)/H(3) receptor antagonists in seasonal allergic rhinitis. Int Arch Allergy Immunol 2012;158:84-98

51. Kiss R, Keseru GM. Histamine H4 receptor ligands and their potential therapeutic applications: an update. Expert Opin Ther Pat 2012;22:205-21

52. Tsabouri S, Tseretopoulou X, Priftis K, Ntzani EE. Omalizumab for the treatment of inadequately controlled allergic rhinitis: a systematic review and meta-analysis of randomized clinical trials. J Allergy Clin Immunol Pract 2014;2:332-40

53. Braido F, Sclifo F, Ferrando M, Canonica GW. New therapies for allergic rhinitis. Curr Allergy Asthma Rep 2014:14:422

54. Grundling C, Schimetta W, Frass M. Real-life effect of classical homeopathy in the treatment of allergies: A multicenter prospective observational study. Wien Klin Wochenschr 2012;124:11-17

55. Brozek JL, Bousquet J, Baena-Cagnani CE, et al. Allergic Rhinitis and its Impact on Asthma (ARIA) guidelines: 2010 revision. J Allergy Clin Immunol 2010;126:466-76

56. Wallace DV, Dykewicz MS, Bernstein DI, et al. The diagnosis and management of rhinitis: an updated practice parameter. J Allergy Clin Immunol 2008;122:S1-84

57. Wong GW, Miravitlles M, Chisholm A, Krishnan JA. Respiratory guidelines-which real world? Ann Am Thorac Soc 2014; 11(Suppl 2):S85-91

- This reference is of particular interest as it highlights limitations inherent in many disease management guidelines.

58. Harbour R, Miller J. A new system for grading recommendations in evidence based guidelines. BMJ 2001;323:334-6

59. Price D, Bateman ED, Chisholm A, et al. Complementing the randomized controlled trial evidence base. Evolution not revolution. Ann Am Thorac Soc 2014; 11(Suppl 2):S92-8

- This reference is of particular interest as it discusses where observational studies and pragmatic trials have utility in addressing clinical questions unanswered and/or unanswerable by randomized controlled trials and advocates a complementary approach where study design aligns with the question asked.

60. Costa DJ, Amouyal M, Lambert P, et al. How representative are clinical study patients with allergic rhinitis in primary care? J Allergy Clin Immunol 2011;127: 920-6

61. Konstantinou GN. Pragmatic trials: how to adjust for the 'Hawthorne effect'? Thorax 2012;67:562

62. Roche N, Reddel HK, Agusti A, et al. Integrating real-life studies in the global therapeutic research framework. Lancet Respir Med 2013;1:e29-30

- This reference is of particular interest as it shows how each type of clinical trial interacts and complements the other, from randomized controlled trials at one end of the spectrum to observational studies at the other end.

63. Rawlins M. De testimonio: on the evidence for decisions about the use of therapeutic interventions. Lancet 2008;372:2152-61

64. Balshem H, Helfand M, Schunemann HJ, et al. GRADE guidelines: 3. Rating the quality of evidence. J Clin Epidemiol 2011;64:401-6

65. Guyatt GH, Oxman AD, Vist GE, et al. GRADE: an emerging consensus on rating quality of evidence and strength of recommendations. BMJ 2008;336:924-6

66. Schunemann HJ, Oxman AD, Brozek J, et al. GRADE: assessing the quality of evidence for diagnostic recommendations. ACP J Club 2008;149:2

67. Roche N, Reddel H, Martin R, et al. Quality standards for real-world research. Focus on observational database studies of comparative effectiveness. Ann Am Thorac Soc 2014;11(Suppl 2):S99-104

-• This reference is of particular interest as it describes quality standards for real-life research. Such high-quality observational studies can then reach a sufficient level of quality to inform guidelines.

68. Vestbo J, Hurd SS, Agusti AG, et al. Global strategy for the diagnosis, management, and prevention of chronic obstructive pulmonary disease: GOLD executive summary. Am J Respir Crit Care Med 2013;187:347-65
69. Bousquet J, Khaltaev N, Cruz AA, et al. Allergic rhinitis and its impact on asthma (ARIA) 2008 update (in collaboration with the world health organization, GA(2)LEN and AllerGen). Allergy 2008;63(Suppl 86): 8-160

70. Scadding GK, Durham SR, Mirakian R, et al. BSACI guidelines for the management of allergic and non-allergic rhinitis. Clin Exp Allergy 2008;38:19-42

71. Schulz KF, Altman DG, Moher D. CONSORT 2010 statement: updated guidelines for reporting parallel group randomised trials. BMJ 2010;340:c332

72. Okubo K, Nakashima M, Miyake N, et al. Comparison of fluticasone furoate and fluticasone propionate for the treatment of Japanese cedar pollinosis. Allergy Asthma Proc 2009;30:84-94

73. Carr W, Bernstein J, Lieberman P, et al. A novel intranasal therapy of azelastine with fluticasone for the treatment of allergic rhinitis. J Allergy Clin Immunol 2012;129: 1282-9

74. Chavannes N, Stallberg B, Lisspers K, et al. UNLOCK: Uncovering and Noting Long-term Outcomes in COPD to enhance knowledge. Prim Care Respir J 2010;19:408

75. Takahashi Y, Nishida Y, Asai S. Utilization of health care databases for pharmacoepidemiology. Eur J Clin Pharmacol 2012;68:123-9

76. Colice G, Martin RJ, Israel E, et al. Asthma outcomes and costs of therapy with extrafine beclomethasone and fluticasone. J Allergy Clin Immunol 2013;132:45-54

77. Kemp L, Haughney J, Barnes N, et al. Cost-effectiveness analysis of corticosteroid inhaler devices in primary care asthma management: A real world observational study. Clinicoecon Outcomes Res 2010;2: 75-85

78. EMA guideline. Guideline on the clinical development of medicinal products for the treatment of allergic rhinoconjunctivitis. Available from: www.emaeuropaed/docs/ en_GB/document_library/ Scientific_guideline/2009/09/ WC500003554.pdf 2004

79. GINA Guidelines. 2014. Available from: www.ginasthma.org/GINA-Report [Last accessed 20 April 15]

80. Nathan RA, Dalal AA, Stanford RH, et al Qualitative development of the rhinitis control assessment test (RCAT), an instrument for evaluating rhinitis symptom control. Patient 2010;3:91-9 
81. Demoly P, Calderon MA, Casale T, et al. Assessment of disease control in allergic rhinitis. Clin Transl Allergy 2013;3:7

82. Fonseca JA, Nogueira-Silva L, Morais-Almeida M, et al. Control of Allergic Rhinitis and Asthma Test (CARAT) can be used to assess individual patients over time. Clin Transl Allergy 2012;2:16

83. Price D, Papadopoulos N, Scadding G, et al. Assessing allergic rhinitis symptom control: results from a digital survey conducted during EAACI 2013. Presented at European Academy of Allergy and
Clinical Immunology Congress, 7 - 11 June 2014, Copenhagen, Denmark

- This reference is of interest as it shows how in real life allergic rhinitis control is assessed in many different ways, and advocates a simple visual analogue scale to assess control and direct treatment in real life.

84. Bousquet PJ, Combescure C, Klossek JM, et al. Change in visual analog scale score in a pragmatic randomized cluster trial of allergic rhinitis. J Allergy Clin Immunol 2009; 123:1349-54

85. Bousquet PJ, Combescure C, Neukirch F, et al. Visual analog scales can assess the severity of rhinitis graded according to ARIA guidelines. Allergy 2007;62:367-72

86. Bousquet J, Bachert C, Canonica GW, et al. Efficacy of desloratadine in persistent allergic rhinitis - a GA(2)LEN study. Int Arch Allergy Immunol 2010;153:395-402

87. Bousquet J, Bachert C, Canonica GW, et al. Efficacy of desloratadine in intermittent allergic rhinitis: a GA(2)LEN study. Allergy 2009;64:1516-23

88. Available from:www.effectivenessevaluation. org

\section{Affiliations}

David Price

Research in Real Life, Singapore, Singapore

Pete Smith

Department of Clinical Medicine, Griffith University, Southport, Australia

Peter Hellings

Department of Otorhinolaryngology, University Hospitals Leuven, Leuven, Belgium

Nikos Papadopoulos

Allergy Department, 2nd Pediatric Clinic, University of Athens, Athens, Greece

Wytske Fokkens

Department of Otorhinolaryngology, Academic Medical Center (AMC) Amsterdam, Amsterdam, The Netherlands

Antonella Muraro

Food Allergy Referral Centre, Veneto Region, Department of Women and Child Health, Padua University Hospital, Padua, Italy

Ruth Murray

Medscript (Scientific \& Medical Affairs), Dundalk, Ireland

\section{Alison Chisholm}

Respiratory Effectiveness Group, Cambridge, UK

Pascal Demoly

Department of Pulmonology - Division of Allergy, University Hospital of Montpellier, Hôpital Arnaud de Villeneuve, France
Glenis Scadding

RNTNE Hospital, London, UK

Joaquim Mullol

Hospital Clinic, IDIBAPS, CIBERES, Barcelona, Catalonia, Spain

Phil Lieberman

University of Tennessee College of Medicine, Memphis, USA

\section{Claus Bachert}

Upper Airways Research Laboratory (URL), University Hospital Ghent, Ghent, Belgium

\section{Ralph Mösges}

Institute of Medical Statistics, Informatics, and Epidemiology, Medical Faculty, University of Köln, Cologne, Germany

\section{Dermot Ryan}

University of Edinburgh, Edinburgh, UK and Optimum Patient Care, Cambridge, UK

\section{Jean Bousquet}

University Hospital, Montpellier, France

and

MACVIA-LR, Contre les Maladies Chronique spour un Vieillissement Actif en Languedoc Roussilon, European Innovation Partnership on Active and Healthy Ageing Reference Site, France

and

INSERM, VIMA: Ageing and chronic diseases. Epidemiological and public health approaches, U1168, Paris, and UVSQ, UMR-S 1168, Universite Versailles St-Quentin-en-Yvelines, France 